\title{
SIMPLIFICATION OF GEOPOTENTIAL PERTURBING FORCE ACTING ON A SATELLITE
}

\author{
M. Eshagh ${ }^{1 *}$, M. Abdollahzadeh ${ }^{2}$ \\ $1{ }^{1}$ Royal Institute of Technology, Division of Geodesy, Stockholm, Sweden \\ ${ }^{2}$ K.N.Toosi University of Technology, Dept. of Geodesy and Geomatics, Tehran, Iran \\ e-mails: eshagh@kth.se,m_abdollahzadeh@sina.kntu.ac.ir \\ M. Najafi-Alamdari \\ K.N.Toosi University of Technology, Dept. of Geodesy and Geomatics, Tehran, Iran \\ e-mail: mnajalm@yahoo.com
}

\begin{abstract}
One of the aspects of geopotential models is orbit integration of satellites. The geopotential acceleration has the largest influence on a satellite with respect to the other perturbing forces. The equation of motion of satellites is a secondorder vector differential equation. These equations are further simplified and developed in this study based on the geopotential force. This new expression is much simpler than the traditional one as it does not derivatives of the associated Legendre functions and the transformations are included in the equations. The maximum degree and order of the geopotential harmonic expansion must be selected prior to the orbit integration purposes. The values of the maximum degree and order of these coefficients depend directly on the satellite's altitude. In this article, behaviour of orbital elements of recent geopotential satellites, such as CHAMP, GRACE and GOCE is considered with respect to the different degree and order of geopotential coefficients. In this case, the maximum degree 116, 109 and 175 were derived for the Earth gravitational field in short arc orbit integration of the CHAMP, GRACE and GOCE, respectively considering millimeter level in perturbations.
\end{abstract}

Keywords: Geopotential, orbit integration, average power acceleration, orbital elements

\section{INTRODUCTION}

The spherical harmonic expansion is a mathematical tool for approximating the Earth's gravitational field. The harmonic coefficients of this expansion can be determined in various ways, say, by using terrestrial and/or satellite data. Only the long wavelength structure of the Earth's gravitational field can be determined by the satellites, because of attenuation of the gravitational signal due to the satellite altitude. Different satellite missions have been dedicated for such aims. The last three satellite missions are CHAMP (Challenging Minisatellite Payload) [Reigber et al., 1999 and 2004], GRACE (Gravity Recovery and Climate Experiments) [Tapley et al., 2005] and upcoming GOCE (Gravity Field and Steady-state Ocean Circulation Explorer) missions [ESA, 1999, Albertella et al., 2002, Balmino et al., 1998 and 2001]. The CHAMP mission was designed based on satellite-to-satellite tracking and analysis of 
the precise orbit of the satellite for recovery of the gravitational field. The GRACE mission is a common project between US and Germany and in this technique low-low mode of the satellite to satellite tracking (SST) are used as space gradiometry. The GOCE mission was dedicated to be launched and in this satellite mission the space gradiometry technique is used as well. The GOCE mission is considered to be the first gradiometric mission although there is a concept for the GRACE satellites as a large one-dimensional gradiometer.

Satellite orbit analysis is a well-known technique for gravitational field recovery cf. e.g., Kaula (1966), Visser (1992), Sneeuw (1992). It is important to consider that although the satellite gradiometry techniques [Rummel et al., 1993, Keller and Sharifi, 2005, Sharifi, 2006] are used in two last missions, the satellite's orbit should be determined as precise as possible so that the extracted perturbations can be analyzed without worrying about biases in the solution. The precise orbit determination (POD) [Su, 2000 and Wolf, 2000] can be done in different ways, such as Kinematic POD, dynamic POD, reduced dynamic POD, etc; see e.g. Rim and Schutz (2001). In this paper we concentrate on orbit integration [Eshagh, 2003a and 2003b] which is the prediction part of the orbit in the dynamic POD. Numerical integration of the orbit has some benefits with respect to analytical one [Kaula, 1966], as it is not restricted to the mathematical models of the perturbing forces. For details of the algorithms; see e.g. Su (2000), Wolf (2000), Eshagh (2003a and 2005) and Sharifi (2006), Eshagh and Najafi-Alamdari (2005a, 2005b, 2006 and 2007).

The equation of motion of satellite is a second-order vector differential equation and the satellite acceleration vector is integrated twice to obtain the vector of velocities and positions. Integrating the equation of motion once yields a velocity vector (and three unknowns), integrating it twice results in a position vector (six unknown integration constant). The traditional expression for the vector differential equation of satellite's motion is complicated as it includes partial derivatives of the spherical coordinates with respect to Cartesian coordinates, derivative of associated Legendre functions (ALF) and singular terms when satellite approaches the poles (near polar satellite). The satellite acceleration is a summation of different gravitational and non-gravitational accelerations, but the largest perturbation is due to the geopotential field. In this study we concentrate on the geopotential force and simplify the vector differential equation of motion of satellite so that it excludes the mentioned complications. This paper continues the previous studies of the authors and also considers the behavior of the orbital elements in each degree of the geopotential field in the recent satellite missions CHAMP, GRACE and GOCE.

In the next section, we present the traditional expression of satellite's equation of motion. In Section 3 we derive a new expression for equation of motion and Section 4 presents an alternative non-singular expression. Section 5 deals with the system of equation to be integrated. In Section 6 the average acceleration power of the gravitational vector is considered to determine maximum degree of geopotential expansion needed for orbit integration. In Section 7 a numerical study on the orbital elements are presented and the paper is ended with conclusions in Section 8. 


\section{TRADITIONAL EXPRESSION OF EQUATION OF MOTION OF}

\section{SATELLITE}

The gravitational field of the Earth can be approximated by a truncated series of spherical harmonics [Heiskanen and Moritz, 1967]:

$$
U(P)=\frac{G M}{R} \sum_{n=0}^{N_{\text {max }}} \sum_{m=-n}^{n}\left(\frac{R}{r}\right)^{n+1} \bar{u}_{n m} Q_{m}(|m| \lambda) \bar{P}_{n|m|}(\cos \theta),(r \geq R)
$$

where, $G M$ is the geocentric constant, $R$ is the radius of the mean sphere of the Earth, $\bar{u}_{n m}$ is the co-sine and sine geopotential coefficient when $m \leq 0$ and $m>0$, respectively. $\bar{P}_{n|m|}$ is the fully-normalized ALF of degree $n$ and order $m, r, \lambda, \theta$ are the spherical Earth-fixed coordinate of the satellite at point $P$ and

$$
Q_{m}(|m| \lambda)= \begin{cases}\cos (|m| \lambda) & m \leq 0 \\ \sin (|m| \lambda) & m>0\end{cases}
$$

Transformations of these triple parameters $r, \lambda, \theta$ to the Cartesian quasi-inertial coordinates of $x, y$, and $\mathrm{z}$ are:

$$
\begin{gathered}
r=\sqrt{x^{2}+y^{2}+z^{2}}, \\
\theta=\pi / 2-\arcsin (z / r), \\
\lambda=\arctan (y / x)-\Theta .
\end{gathered}
$$

where $\Theta$ is Greenwich apparent sidereal time. The partial derivatives of these curvilinear and Cartesian coordinates is:

$$
\begin{gathered}
\partial r / \partial r_{i}=r_{i} / r \\
\frac{\partial \theta}{\partial r_{i}}=-\left(x^{2}+y^{2}\right)^{-1 / 2}\left[-z r_{i} / r^{2}+\partial z / \partial r_{i}\right], \\
\frac{\partial \lambda}{\partial r_{i}}=x\left(y^{2}+x^{2}\right)^{-1}\left[\partial y / \partial r_{i}-y / x \times \partial x / \partial r_{i}\right],
\end{gathered}
$$

where, $r_{i}, i=1,2,3$ stand for $x, y$ and $z$, respectively. Now the derivatives of potential $U(P)$ with respect to spherical coordinates must be derived. These derivatives are well-known for details see, e.g. Parrot (1989), Santos (1994) and Hwang and Lin, (1998). According to Hwang and Lin (1998) the satellite acceleration can also be expressed in the quasi-inertial frame using the following transformation (neglecting precession, nutation and polar motion but for actual orbit analysis one cannot neglect these effects): 


$$
\left[\begin{array}{l}
\ddot{x}(P) \\
\ddot{y}(P) \\
\ddot{z}(P)
\end{array}\right]=\left[\begin{array}{ccc}
\frac{x}{r} & \frac{x z}{r \sqrt{x^{2}+y^{2}}} & \frac{-y}{\sqrt{x^{2}+y^{2}}} \\
\frac{y}{r} & \frac{y z}{r \sqrt{x^{2}+y^{2}}} & \frac{x}{\sqrt{x^{2}+y^{2}}} \\
\frac{z}{r} & \frac{-\sqrt{x^{2}+y^{2}}}{r} & 0
\end{array}\right]\left[\begin{array}{c}
\frac{\partial U(P)}{\partial r} \\
\frac{\partial U(P)}{r \partial \theta} \\
\frac{\partial U(P)}{r \sin \theta \theta \lambda}
\end{array}\right]
$$

The traditional expressions for the elements of gravitational vector in geocentric Earth-fixed frame are:

$$
\begin{aligned}
& \frac{\partial U(P)}{\partial r}=-\frac{G M}{R^{2}} \sum_{n=0}^{N_{\max }}(n+1)\left(\frac{R}{r}\right)^{n+2} \sum_{m=-n}^{n} \bar{u}_{n m} Q_{m}(|m| \lambda) \bar{P}_{n|m|}(\cos \theta) \\
& \frac{\partial U(P)}{r \partial \theta}=\frac{G M}{R^{2}} \sum_{n=0}^{N_{\max }} \sum_{m=-n}^{n}\left(\frac{R}{r}\right)^{n+2} \bar{u}_{n m} Q_{m}(|m| \lambda) \frac{\partial \bar{P}_{n|m|}(\cos \theta)}{\partial \theta} \\
& \frac{\partial U(P)}{r \sin \theta \partial \lambda}=\frac{G M}{R^{2}} \sum_{n=0}^{N_{\max }}\left(\frac{R}{r}\right)^{n+2} \sum_{m=-n}^{n} m \bar{u}_{n m} Q_{-m}(|m| \lambda) \frac{\bar{P}_{n|m|}(\cos \theta)}{\sin \theta} .
\end{aligned}
$$

Non-singular expressions for the elements of the gravitational vector are given by Eshagh (2008a). Equation (5) is the differential equation of satellite's motion. This equation is involved with derivatives of the curvilinear coordinates. Therefore the second-order derivatives of the gravitational potential will have a complicated form for each element of the acceleration vector. In the following section an attempt is made to simplify this differential equation further.

\section{NEW EXPRESSIONS FOR EQUATION OF MOTION OF}

\section{SATELLITE}

As it was shown in previous section, to carry out the integration steps of satellite orbit in the quasi-inertial frame while the geopotential model (e.g., EGM96, Lemoine et al., 1996) as the source of geopotential is given in an Earth fixed (non-inertial) curvilinear coordinates system, one has to transfer the satellite position from the inertial frame to the Earth fixed frame, for the acceleration computation out of the Earth gravitational model. This requires the Cartesian and Curvilinear coordinates of the satellite to be computed in both the quasi-inertial and the Earth fixed frames.

Similar to the previous section we can write the following transformation for the satellite's accelerations in a quasi-inertial frame, but this time we change the transformation matrix from Cartesian to curvilinear form: 


$$
\left[\begin{array}{l}
\ddot{x}(P) \\
\ddot{y}(P) \\
\ddot{z}(P)
\end{array}\right]=\left[\begin{array}{ccc}
\sin \theta \cos \lambda^{*} & \cos \lambda^{*} \cos \theta & -\sin \lambda^{*} \\
\sin \theta \sin \lambda^{*} & \sin \lambda^{*} \cos \theta & \cos \lambda^{*} \\
\cos \theta & -\sin \theta & 0
\end{array}\right]\left[\begin{array}{c}
\frac{\partial U(P)}{\partial r} \\
\frac{\partial U(P)}{r \partial \theta} \\
\frac{\partial U(P)}{r \sin \theta \theta \lambda}
\end{array}\right]
$$

where $\lambda^{*}=\lambda+\Theta$ as defined previously. By inserting the components of the gravitational vector Eqs. (6a)-(6c) into the Eq. (7) and after simplifications we obtain :

$$
\begin{aligned}
& \ddot{x}(P)=\frac{G M}{R^{2}} \sum_{n=0}^{N_{\max }}\left(\frac{R}{r}\right)^{n+2} \sum_{m=-n}^{n} \bar{u}_{n m}\left[\cos \lambda^{*} Q_{m}(|m| \lambda) F_{n m}(\theta)-\sin \lambda^{*} Q_{-m}(|m| \lambda) G_{n m}(\theta)\right] \\
& \ddot{y}(P)=\frac{G M}{R^{2}} \sum_{n=0}^{N_{\max }}\left(\frac{R}{r}\right)^{n+2} \sum_{m=-n}^{n} \bar{u}_{n m}\left[\sin \lambda^{*} Q_{m}(|m| \lambda) F_{n m}(\theta)-\cos \lambda^{*} Q_{-m}(|m| \lambda) G_{n m}(\theta)\right] \\
& \ddot{z}(P)=-\frac{G M}{R^{2}} \sum_{n=0}^{N_{\max }}\left(\frac{R}{r}\right)^{n+2} \sum_{m=-n}^{n} \bar{u}_{n m} Q_{m}(|m| \lambda)\left[(n+1) \cos \theta \bar{P}_{n|m|}+\sin \theta \frac{\partial \bar{P}_{n|m|}}{\partial \theta}\right]
\end{aligned}
$$

where

$$
\begin{aligned}
& F_{n m}(\theta)=-(n+1) \sin \theta \bar{P}_{n|m|}+\cos \theta \frac{\partial \bar{P}_{n|m|}}{\partial \theta} \\
& G_{n m}(\theta)=m \frac{\bar{P}_{n|m|}}{\sin \theta}=f_{n m} \bar{P}_{n-1,|m|-1}+g_{n m} \bar{P}_{n-1,|m|+1}|m| \neq 0 \\
& f_{n m}=\frac{m}{2|m|} \sqrt{n+|m|} \sqrt{n+|m|-1} \sqrt{\frac{\left(2-\delta_{|m| 0}\right)(2 n+1)}{\left(2-\delta_{|m|-1,0}\right)(2 n-1)}} \\
& g_{n m}=\frac{m}{2|m|} \sqrt{n-|m|} \sqrt{n-|m|-1} \sqrt{\frac{\left(2-\delta_{|m| 0}\right)(2 n+1)}{\left(2-\delta_{|m|+1,0}\right)(2 n-1)}}
\end{aligned}
$$

Equations (9b)-(9d) were given by Eshagh (2008a, Proposition 3), $f_{n m}$ and $g_{n m}$ are derived based on normalizing Lemma 5 using Eq. (11), which will be presented later. It should be mentioned that the order $m$ is a part of $f_{n m}, g_{n m}, F_{n m}(\theta)$ and $G_{n m}(\theta)$. Now an attempt is made to simplify these coefficients further. For presenting our mathematical derivations we define the following lemmas.

Lemma 1 [Ilk 1983, Eq. (Z. 1.43)]:

$$
\sin \theta \frac{\partial P_{n|m|}}{\partial \theta}=-\frac{(n+1)(n+|m|)}{(2 n+1)} P_{n-1,|m|}+\frac{n(n-|m|+1)}{(2 n+1)} P_{n+1,|m|}
$$

Lemma 2 [Ilk 1983, Eq. (Z.1.37)]: 


$$
\cos \theta P_{n|m|}=\frac{n+|m|}{2 n+1} P_{n-1,|m|}+\frac{n-|m|+1}{2 n+1} P_{n+1,|m|} .
$$

Note: these relations only hold for non-normalized ALF.

\section{Proposition 1:}

$$
(n+1) \cos \theta \bar{P}_{n|m|}+\sin \theta \frac{\partial \bar{P}_{n|m|}}{\partial \theta}=\sqrt{n-|m|+1} \sqrt{n+|m|+1} \sqrt{\frac{2 n+1}{2 n+3}} \bar{P}_{n+1,|m|}
$$

Proof. By multiplying Lemma 2 by $(n+1)$ and adding to Lemma 1 we obtain :

$$
(n+1) \cos \theta P_{n|m|}+\sin \theta \frac{\partial P_{n|m|}}{\partial \theta}=(n-|m|+1) P_{n+1,|m|},
$$

by considering normalization factors

$$
P_{n|m|}=\sqrt{\frac{(n+|m|) !}{\left(2-\delta_{|m| 0}\right)(2 n+1)(n-|m|) !}} \bar{P}_{n|m|} .
$$

By normalizing Eq. (10) based on Eq. (11) and after further simplifications Proposition 1 is proved.

Corollary 1 The satellite acceleration in z-direction in the quasi-inertial frame is:

$$
\ddot{z}(P)=-\frac{G M}{R^{2}} \sum_{n=0}^{N_{\max }}\left(\frac{R}{r}\right)^{n+2} \sum_{m=-n}^{n} \bar{u}_{n m} Q_{m}(|m| \lambda) \sqrt{n-|m|+1} \sqrt{n+|m|+1} \sqrt{\frac{2 n+1}{2 n+3}} \bar{P}_{n+1,|m|}
$$

Here, the result follows by considering and re-substituting the results of Proposition 1 into Eq. (8c).

For simplifying the co-latitude dependent parts of Eqs. (8a) and (8b), we start our mathematical derivations by the following lemmas.

Lemma 3 [Ilk 1983, Eq. (Z.1.44)]:

$$
\frac{\partial P_{n|m|}}{\partial \theta}=\frac{1}{2}\left[(n+|m|)(n-|m|+1) P_{n,|m|-1}-P_{n,|m|+1}\right]
$$

Lemma 4 [Ilk 1983, Eq. (Z.1.40)]:

$$
\sin \theta P_{n|m|}=-\frac{1}{2 n+1} P_{n-1,|m|+1}+\frac{1}{2 n+1} P_{n+1,|m|+1}
$$

Lemma 5 [Ilk 1983, Eq. (Z.1.42)]: 


$$
\frac{P_{n|m|}}{\sin \theta}=\frac{1}{2|m|}\left[(n+|m|)(n+|m|-1) P_{n-1,|m|-1}+P_{n-1,|m|+1}\right] .
$$

Note: all relations hold for non-normalized ALF.

\section{Proposition 2:}

$$
\cos \theta \frac{\partial \bar{P}_{n|m|}}{\partial \theta}-(n+1) \sin \theta \bar{P}_{n|m|}=a_{n m} \bar{P}_{n-1,|m|-1}+b_{n m} \bar{P}_{n-1,|m|+1}+c_{n m} \bar{P}_{n+1,|m|-1}+d_{n m} \bar{P}_{n+1,|m|+1}
$$

where

$$
\begin{gathered}
a_{n m}=\frac{n-|m|}{2} \sqrt{\frac{\left(2-\delta_{|m| 0}\right)(n+|m|)(n+|m|-1)}{\left(2-\delta_{|m|-1,0}\right)(2 n+1)(2 n-1)}} \\
b_{n m}=\frac{n-|m|}{2} \sqrt{\frac{\left(2-\delta_{|m| 0}\right)(n-|m|)(n-|m|-1)}{\left(2-\delta_{|m|+1,0}\right)(2 n+1)(2 n-1)}} \\
c_{n m}=\frac{n+|m|+1}{2} \sqrt{\frac{\left(2-\delta_{|m| 0}\right)(n-|m|+1)(n-|m|+2)}{\left(2-\delta_{|m|-1,0}\right)(2 n+1)(2 n+3)}} \\
d_{n m}=-\frac{3 n-|m|+1}{2} \sqrt{\frac{\left(2-\delta_{|m| 0}\right)(n+|m|+1)(n+|m|+2)}{\left(2-\delta_{|m|+1,0}\right)(2 n+1)(2 n+3)}}
\end{gathered}
$$

Proof. We have to derive a relation for $\cos \theta \frac{\partial P_{n|m|}}{\partial \theta}$. In this case let us differentiate Lemma 2 with respect to $\theta$ (co-latitude)

$$
-\sin \theta P_{n|m|}+\cos \theta \frac{\partial P_{n|m|}}{\partial \theta}=\frac{n+|m|}{2 n+1} \frac{\partial P_{n-1, m}}{\partial \theta}+\frac{n-|m|+1}{2 n+1} \frac{\partial P_{n+1, m}}{\partial \theta}
$$

considering Lemma 3 for the derivatives of the unnormalized ALF and inserting them in Eq. (12) we finally obtain

$$
\begin{aligned}
-\sin \theta P_{n|m|}+\cos \theta \frac{\partial P_{n|m|}}{\partial \theta} & =\frac{n+|m|}{2(2 n+1)}\left[(n+|m|-1)(n-|m|) P_{n-1,|m|-1}-P_{n-1,|m|+1}\right]+ \\
+ & \frac{n-|m|+1}{2(2 n+1)}\left[(n+|m|+1)(n-|m|+2) P_{n+1,|m|-1}-P_{n+1,|m|+1}\right] .
\end{aligned}
$$

by taking $-\sin \theta P_{n|m|}$ into the right hand side of Eq. (13) and considering Lemma 4 we obtain: 


$$
\begin{aligned}
\cos \theta \frac{\partial P_{n|m|}}{\partial \theta} & =\frac{(n+|m|)(n+|m|-1)(n-|m|)}{2(2 n+1)} P_{n-1,|m|-1}-\frac{n+|m|+2}{2(2 n+1)} P_{n-1,|m|+1}+ \\
& +\frac{(n-|m|+1)(n+|m|+1)(n-|m|+2)}{2(2 n+1)} P_{n+1,|m|-1}-\frac{n-|m|-1}{2(2 n+1)} P_{n+1,|m|+1} .
\end{aligned}
$$

Multiplying Lemma 4 by $-(n+1)$ and adding to Eq. (14) we have

$$
\begin{gathered}
\cos \theta \frac{\partial P_{n|m|}}{\partial \theta}-(n+1) \sin \theta P_{n|m|}=\frac{(n+|m|)(n+|m|-1)(n-|m|)}{2(2 n+1)} P_{n-1,|m|-1}+\frac{n-|m|}{2(2 n+1)} P_{n-1,|m|+1}+ \\
+\frac{(n-|m|+1)(n+|m|+1)(n-|m|+2)}{2(2 n+1)} P_{n+1,|m|-1}-\frac{3 n-|m|+1}{2(2 n+1)} P_{n+1,|m|+1}
\end{gathered}
$$

The proposition is proved by normalizing Eq. (15) and considering Eq. (11) as the normalizing factor.

Corollary 2 The satellite acceleration in $x$ - and $y$-directions of the quasi-inertial frame are:

$$
\begin{aligned}
\ddot{x}(P) & =\frac{G M}{R^{2}} \sum_{n=0}^{N_{\max }}\left(\frac{R}{r}\right)^{n+2} \sum_{m=-n}^{n} \bar{u}_{n m}\left[\operatorname { c o s } \lambda ^ { * } Q _ { m } ( | m | \lambda ) \left(a_{n m} \bar{P}_{n-1,|m|-1}+b_{n m} \bar{P}_{n-1,|m|+1}+\right.\right. \\
& \left.\left.+c_{n m} \bar{P}_{n+1,|m|-1}+d_{n m} \bar{P}_{n+1,|m|+1}\right)-\sin \lambda^{*} Q_{-m}(|m| \lambda)\left(f_{n m} \bar{P}_{n-1,|m|-1}+g_{n m} \bar{P}_{n-1,|m|+1}\right)\right] \\
\ddot{y}(P) & =\frac{G M}{R^{2}} \sum_{n=0}^{N_{\max }}\left(\frac{R}{r}\right)^{n+2} \sum_{m=-n}^{n} \bar{u}_{n m}\left[\operatorname { s i n } \lambda ^ { * } Q _ { m } ( | m | \lambda ) \left(a_{n m} \bar{P}_{n-1,|m|-1}+b_{n m} \bar{P}_{n-1,|m|+1}+\right.\right. \\
& \left.\left.+c_{n m} \bar{P}_{n+1,|m|-1}+d_{n m} \bar{P}_{n+1,|m|+1}\right)-\cos \lambda^{*} Q_{-m}(|m| \lambda)\left(f_{n m} \bar{P}_{n-1,|m|-1}+g_{n m} \bar{P}_{n-1,|m|+1}\right)\right]
\end{aligned}
$$

The corollary follows by inserting Proposition 2 into Eqs. (8a) and (8b) and considering Eqs. (9a) and (9b). It should be emphasized that $f_{n m}$ and $g_{n m}$ are derived based on normalizing Lemma 5 using Eq. (11), see e.g. Eshagh (2008a, Proposition $3)$.

Note: For the zonal terms of satellite accelerations presented in Corollary 2, $G_{n m}(\theta)=0$, i.e. the second term in both equations of Corollary 2 will vanish.

We have presented the elements of the satellite acceleration vector in Corollaries 1 and 2. It is obvious that the presented formulas are very simple to use as there is neither first- and/or second-order derivatives of the ALF nor singular terms. Also the formulation inherently includes necessary transformation. The satellite's acceleration in $x$ - and $y$-directions, which were presented in Corollary 2 can also be further simplified. In the following section we present alternative formulas for these accelerations. 
4. ALTERNATIVE NON-SINGULAR EXPRESSIONS FOR EQUATION OF MOTION OF SATELLITE

In the previous section we obtained non-singular expressions for the satellite's accelerations in a quasi-inertial frame. We can also further simplify the relations presented in Corollary 2 considering some trigonometric simplifications. The result of these simplifications is:

$$
\begin{aligned}
& \ddot{x}(P)=\frac{G M}{2 R^{2}} \sum_{n=0}^{N_{\max }}\left(\frac{R}{r}\right)^{n+2} \sum_{m=-n}^{n} \bar{u}_{n m}\left[Q_{-m}(\lambda(1+|m|)+\Theta)+\frac{m}{|m|} Q_{m}(\lambda(1-|m|)+\Theta)\right] \\
& \ddot{x}(P)=\frac{G M}{R^{2}} \sum_{n=0}^{N_{\max }}\left(\frac{R}{r}\right)^{n+2} \bar{u}_{n 0} \cos \lambda^{*} F_{n 0}(\theta), \quad \text { for } \quad m=0, \\
& \ddot{y}(P)=\frac{G M}{2 R^{2}} \sum_{n=0}^{N_{\max }}\left(\frac{R}{r}\right)^{n+2} \sum_{m=-n}^{n} \bar{u}_{n m}\left[Q_{m}(\lambda(1-|m|)+\Theta)-\frac{m}{|m|} Q_{n m}(\theta)\right), \text { for } m \neq 0, \\
& \ddot{y}(P)=\frac{G M}{R^{2}} \sum_{n=0}^{N_{\max }}\left(\frac{R}{r}\right)^{n+2} \bar{u}_{n 0} \sin \lambda^{*} F_{n 0}(\theta), \quad \text { for } \quad m=0 .
\end{aligned}
$$

It should be mentioned again that $G_{n m}(\theta)=0$ for the zonal terms and Eqs. (16a)-(16d) can further be simplified. We have added $m /|m|$ in our formulation in Eq. (16b) to keep the right sign for $Q_{m}(\lambda(1+|m|)+\Theta)$, when $m$ is negative a minus sign (-) appears by multiplication of -1 to this coefficient, and when it becomes positive, a plus sign $(+)$ appears instead.

Equations (16a) and (16d) contain two terms $\left(F_{n m}(\theta)-G_{n m}(\theta)\right)$ and $\left(F_{n m}(\theta)+G_{n m}(\theta)\right)$, when $m<0$ and $m>0$, respectively, and they are simplified in the following propositions.

\section{Proposition 3:}

$\cos \theta \frac{\partial \bar{P}_{n|m|}}{\partial \theta}-(n+1) \sin \theta \bar{P}_{n|m|}+m \frac{\bar{P}_{n|m|}}{\sin \theta}=a_{n m}^{\prime} \bar{P}_{n-1,|m|-1}+b_{n m}^{\prime} \bar{P}_{n-1,|m|+1}+c_{n m}^{\prime} \bar{P}_{n+1,|m|-1}+d_{n m}^{\prime} \bar{P}_{n+1,|m|+1}$ where

$$
a_{n m}^{\prime}=\frac{1}{2}\left[n-|m|+\frac{m}{|m|}(2 n+1)\right] \sqrt{\frac{\left(2-\delta_{|m| 0}\right)(n+|m|)(n+|m|-1)}{\left(2-\delta_{|m|-1,0}\right)(2 n+1)(2 n-1)}}
$$




$$
\begin{aligned}
& b_{n m}^{\prime}=\frac{1}{2}\left[n-|m|+\frac{m}{|m|}(2 n+1)\right] \sqrt{\frac{\left(2-\delta_{|m| 0}\right)(n-|m|)(n-|m|-1)}{\left(2-\delta_{|m|+1,0}\right)(2 n+1)(2 n-1)}} \\
& c_{n m}^{\prime}=\frac{n+|m|+1}{2} \sqrt{\frac{\left(2-\delta_{|m| 0}\right)(n-|m|+1)(n-|m|+2)}{\left(2-\delta_{|m|-1,0}\right)(2 n+1)(2 n+3)}} \\
& d_{n m}^{\prime}=-\frac{3 n-|m|+1}{2} \sqrt{\frac{\left(2-\delta_{|m| 0}\right)(n+|m|+1)(n+|m|+2)}{\left(2-\delta_{|m|+1,0}\right)(2 n+1)(2 n+3)}} .
\end{aligned}
$$

Proof. By considering Eq. (15) and Lemma 5 we obtain after simplification:

$$
\begin{aligned}
\cos \theta \frac{\partial P_{n|m|}}{\partial \theta} & -(n+1) \sin \theta P_{n|m|}+m \frac{P_{n|m|}}{\sin \theta}=\frac{(n+|m|)(n+|m|-1)}{2}\left[\frac{n-|m|}{(2 n+1)}+\frac{m}{|m|}\right] P_{n-1,|m|-1}+ \\
+ & \frac{1}{2}\left[\frac{n-|m|}{(2 n+1)}+\frac{m}{|m|}\right] P_{n-1,|m|+1}+\frac{(n-|m|+1)(n+|m|+1)(n-|m|+2)}{2(2 n+1)} P_{n+1,|m|-1}- \\
& -\frac{3 n-|m|+1}{2(2 n+1)} P_{n+1,|m|+1}
\end{aligned}
$$

and the proposition is proved by normalizing Eq. (17) using Eq. (11).

\section{Proposition 4:}

$\cos \theta \frac{\partial \bar{P}_{n|m|}}{\partial \theta}-(n+1) \sin \theta \bar{P}_{n|m|}-m \frac{\bar{P}_{n|m|}}{\sin \theta}=a_{n m}^{\prime \prime} \bar{P}_{n-1,|m|-1}+b_{n m}^{\prime \prime} \bar{P}_{n-1,|m|+1}+c_{n m}^{\prime} \bar{P}_{n+1,|m|-1}+d_{n m}^{\prime} \bar{P}_{n+1,|m|+1}$

where

$$
\begin{aligned}
& a_{n m}^{\prime \prime}=\frac{1}{2}\left[n-|m|-\frac{m}{|m|}(2 n+1)\right] \sqrt{\frac{\left(2-\delta_{|m| 0}\right)(n+|m|)(n+|m|-1)}{\left(2-\delta_{|m|-1,0}\right)(2 n+1)(2 n-1)}} \\
& b_{n m}^{\prime \prime}=\frac{1}{2}\left[n-|m|-\frac{m}{|m|}(2 n+1)\right] \sqrt{\frac{\left(2-\delta_{|m| 0}\right)(n-|m|)(n-|m|-1)}{\left(2-\delta_{|m|+1,0}\right)(2 n+1)(2 n-1)}}
\end{aligned}
$$

Proof. Again by considering Eq. (15) and Lemma 5 and after simplification we obtain: 


$$
\begin{aligned}
\cos \theta \frac{\partial P_{n|m|}}{\partial \theta} & -(n+1) \sin \theta P_{n|m|}+m \frac{P_{n|m|}}{\sin \theta}=\frac{(n-|m|)(n-|m|-1)}{2}\left[\frac{n-|m|}{(2 n+1)}-\frac{m}{|m|}\right] P_{n-1,|m|-1}+ \\
+ & \frac{1}{2}\left[\frac{n-|m|}{(2 n+1)}-\frac{m}{|m|}\right] P_{n-1,|m|-1}+\frac{(n-|m|+1)(n+|m|+1)(n-|m|+2)}{2(2 n+1)} P_{n+1,|m|-1}- \\
& -\frac{3 n-|m|+1}{2(2 n+1)} P_{n+1,|m|+1}
\end{aligned}
$$

and the proposition is proved by normalizing Eq. (18) using Eq. (11).

Note: For $m=0$ terms the third term in the first square bracket of the $a_{n m}^{\prime}, b_{n m}^{\prime}, a_{n m}^{\prime \prime}$ and $b_{n m}^{\prime \prime}$ coefficients presented in Propositions 3 and 4 vanish. In other words, $a_{n 0}^{\prime \prime}=a_{n 0}^{\prime}=a_{n 0}$ and $b_{n 0}^{\prime \prime}=b_{n 0}^{\prime}=b_{n 0}$.

Corollary 3 The satellite acceleration in $x$ - and $y$-directions of the quasi-inertial frame based on Eqs. (16a) and (16b) and Propositions 3 and 4 are:

$$
\begin{array}{r}
\ddot{x}(P)=\frac{G M}{2 R^{2}} \sum_{n=0}^{N_{\text {max }}}\left(\frac{R}{r}\right)^{n+2} \sum_{m=-n}^{n} \bar{u}_{n m}\left[Q_{-m}(\lambda(1+|m|)+\Theta)+\frac{m}{|m|} Q_{m}(\lambda(1-|m|)+\Theta)\right] \\
\left(a_{n m}^{\prime} \bar{P}_{n-1,|m|-1}+b_{n m}^{\prime} \bar{P}_{n-1,|m|+1}+\frac{m}{|m|} c_{n m}^{\prime} \bar{P}_{n+1,|m|-1}+\frac{m}{|m|} d_{n m}^{\prime} \bar{P}_{n+1,|m|+1}\right), m \neq 0 \\
\ddot{y}(P)=\frac{G M}{2 R^{2}} \sum_{n=0}^{N_{\max }}\left(\frac{R}{r}\right)^{n+2} \sum_{m=-n}^{n} \bar{u}_{n m}\left[Q_{m}(\lambda(1-|m|)+\Theta)-\frac{m}{|m|} Q_{-m}(\lambda(1+|m|)+\Theta)\right] \\
\left(a_{n m}^{\prime} \bar{P}_{n-1,|m|-1}+b_{n m}^{\prime} \bar{P}_{n-1,|m|+1}+\frac{m}{|m|} c_{n m}^{\prime} \bar{P}_{n+1,|m|-1}+\frac{m}{|m|} d_{n m}^{\prime} \bar{P}_{n+1,|m|+1}\right), m \neq 0 .
\end{array}
$$

The advantages of our new expressions are to exclude singular terms, derivatives of the ALF and simplicity because of having same type of the coordinates for the same point at satellite level. All the coefficients of the ALF $\left(a_{n m}^{\prime}, b_{n m}^{\prime}, c_{n m}^{\prime}, d_{n m}^{\prime}, a_{n m}^{\prime \prime}, b_{n m}^{\prime \prime}, c_{n m}^{\prime \prime}\right.$ and $\left.d_{n m}^{\prime \prime}\right)$ are constant and do not change by satellite's position. The ALF are needed to be computed once for each position of the satellite.

\section{NUMERICAL ORBIT INTEGRATION}

Equation (5) shows the three differential equations to be solved. Numerical integration is the simplest and most efficient technique for the solution. The RungeKutta [Babolian and Maleknejad, 1994, Eshagh 2003a, Eshagh and Najafi Alamdari, $2006]$ is one of the well-known single step methods of numerical integration. AdamsBashforth and Adams-Moulton [Babolian and Maleknejad, 1994], also StörmerCowell [Santos, 1994] algorithms are two well-known methods of multi-step integration. Step-variable methods can also be used for integration; see Eshagh (2005). The orbit integration in different frames is given by Eshagh (2009b). Some details for the benefits of the reader about orbit integration are given in Appendix A. 


\section{AVERAGE POWER OF ACCELERATION AT SATELLITE ALTITUDE}

Depending on the satellite's altitude, the maximum degree $N_{\text {max }}$, in the spherical harmonic expansion is considered as a cut-off degree. This is due to the factor $(R / r)^{2 n}$ in these equations that attenuates the magnitude of the satellite acceleration and due to the asymptotic decrease in $\bar{u}_{n m}$ coefficients. The average power of the satellite's acceleration was investigated by Hwang and Lin (1998). This power can be written in terms of the degree variance of the gravitational field as [Hwang and Lin, 1998]:

$$
P_{k}=\left(\frac{G M}{R^{\prime 2}}\right)^{2} \sum_{n=2}^{k}\left(\frac{R}{R^{\prime}}\right)^{2 n}(n+1)(2 n+1) \sum_{m=-n}^{n} \bar{u}_{n m}^{2},
$$

where $R^{\prime}$ is approximately equal to the Earth's mean radius plus the satellite mean altitude. With specific error tolerance the cutoff degree can be determined by comparing the power of acceleration up to the cutoff degree and the "total" power of acceleration which can be obtained by an expansion to a very high degree $N_{\max }$. Currently equal to 2160 is the highest possible degree but at satellite level such high degree geopotential model does not make sense. In order to determine the highest useful degree one may compute the relative power up to degree $k$

$$
\rho_{k}=\frac{P_{k}}{P_{N_{\max }}}
$$

we should have $\rho_{k} \approx 1$ or $\left(1-\rho_{k}\right)<10^{-14}$ for the $k$ to be the cut-off degree.

Based on the average acceleration power of the satellite, Eq. (19), and the criterion mentioned above (Eq. 20), the cut-off degree of the spherical harmonic expansion of the gravitational field for integrating the orbit of the CHAMP, GRACE and GOCE satellites are obtained 154, 137 and 261, respectively.

\section{NUMERICAL ESTIMATION OF SATELLITE ORBIT}

The perturbations of the orbital elements are enlarged by decreasing the satellite's elevation. The approximate magnitude of these orbital elements for the recent satellite missions are presented in Table 1.

Table 1. Orbital characteristics of the recent satellite missions, CHAMP, GRACE and upcoming GOCE

\begin{tabular}{|c|c|c|c|}
\hline $\begin{array}{c}\text { Orbital } \\
\text { elements }\end{array}$ & CHAMP & GRACE & GOCE \\
\hline $\mathrm{a}$ (metre) & 6823287 & 6882043 & 6628281 \\
\hline $\mathrm{e}$ & $<0.004$ & $<0.005$ & $<0.001$ \\
\hline $\mathrm{i}$ (deg.) & 87.3 & 89.05 & 96.6 \\
\hline$\Omega$ (deg.) & 144 & 74.51 & 0 \\
\hline$\omega$ (deg.) & 257 & 68.35 & 0 \\
\hline
\end{tabular}


The GRACE twin satellites have higher altitudes than the others, its orbit is more polar as its inclination is closer to $90 \mathrm{deg}$. The GOCE satellite has the most circular and inclined orbit. The current orbital elements of the CHAMP and the GRACE are available on http://www.gfz-potsdam.de/pb1/op/champ/ and http://www.csr.utexas.edu/grace/, respectively. Also the initial position and velocity of the GOCE were received by authors from ESA (European Space Agency) (Plank, personal communication) and converted to the orbital elements.

In order to study the behaviour of orbital elements due to different harmonics we need to integrate Corollaries 1 and either 2 or 3 numerically. Among the various techniques of solving the vector system of differential equations, the well-known 4-th order Runge-Kutta integrator was used in this study because of its simplicity.

The computational speed on the orbit integration process is directly related to the computation of the satellite accelerations due to different resolutions of the gravitational field. A double summation can be summed up using two loops in computer programming, but it is not the best way, in computational point of view by increasing maximum degree of the spherical harmonic expansion computational time is increased too. In such cases, vectorization techniques are preferred. Sharifi (2006) and Eshagh (2009a) have also used this technique for global synthesis and analysis of the Earth gravitational field successfully.

We consider one day revolution of the CHAMP, GRACE and GOCE satellites to compare the behaviour of their orbital elements at each degree of the gravitational field. At first step maximum degree $N_{\max }$ of the spherical harmonic coefficients of the Earth gravitational field were determined for each satellite using average accelerations power presented in Section 5. The integration performs $N_{\max }$ times for computing the satellite state vector in each step. The state vector is converted to the orbital elements and the orbital elements due to the central (spherical) gravitational field are subtracted to obtain the perturbations. The following figures show the behaviour of orbital elements for each satellite with respect to different harmonic degree of gravitational field. In these figures we consider the maximum absolute value of the geopotential perturbation for a specific degree along the satellite motion. 

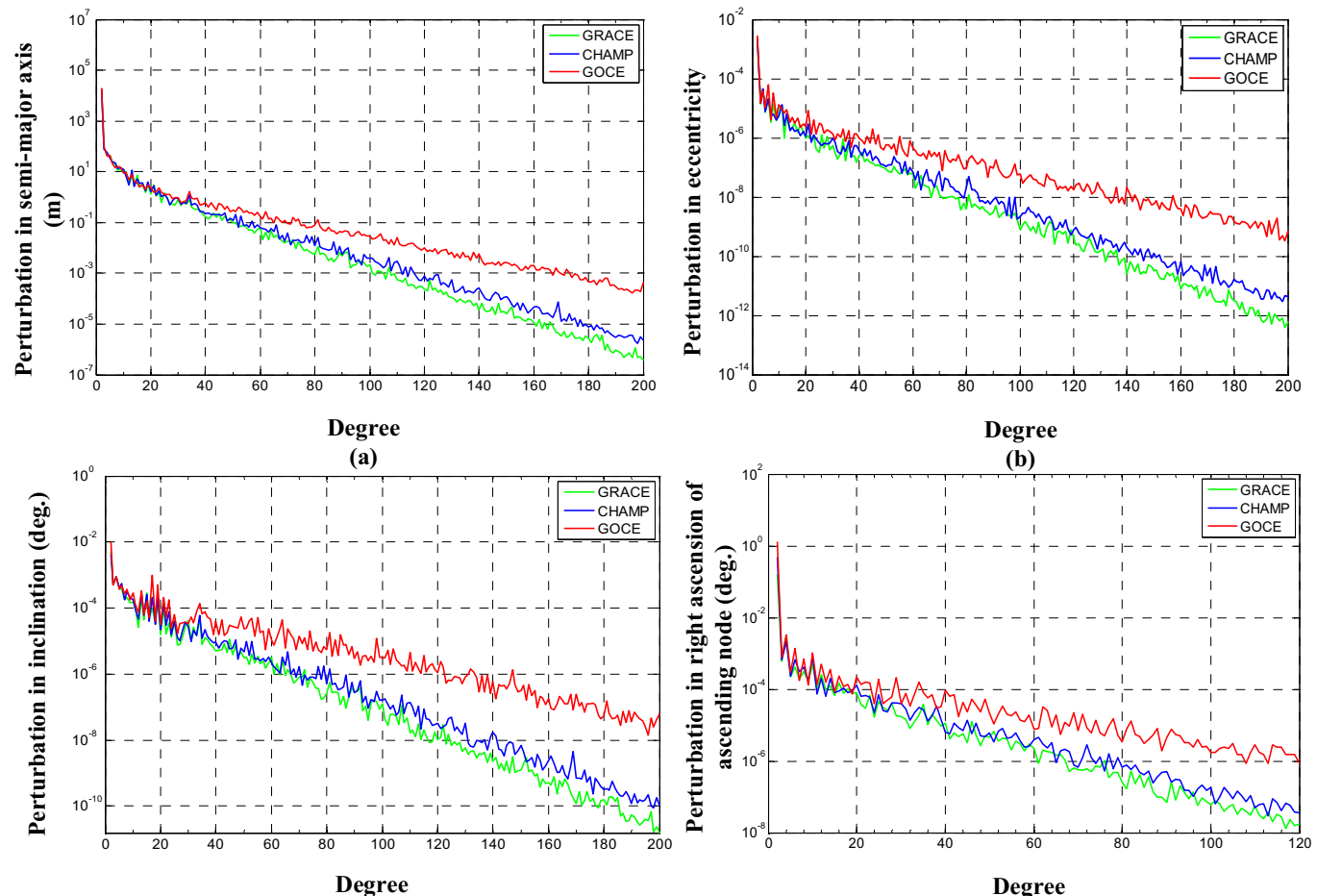

(c)

(d)

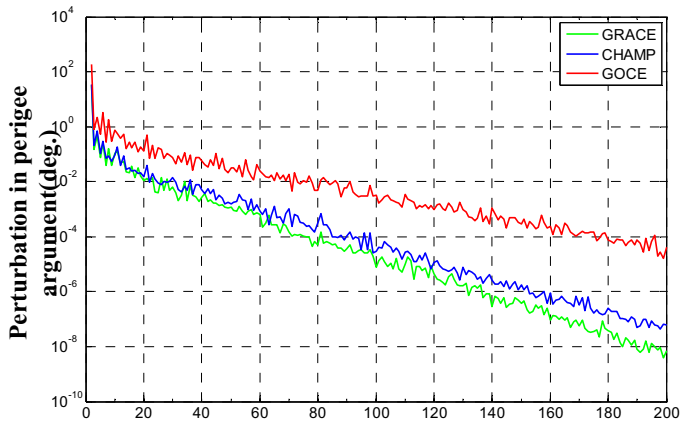

Degree

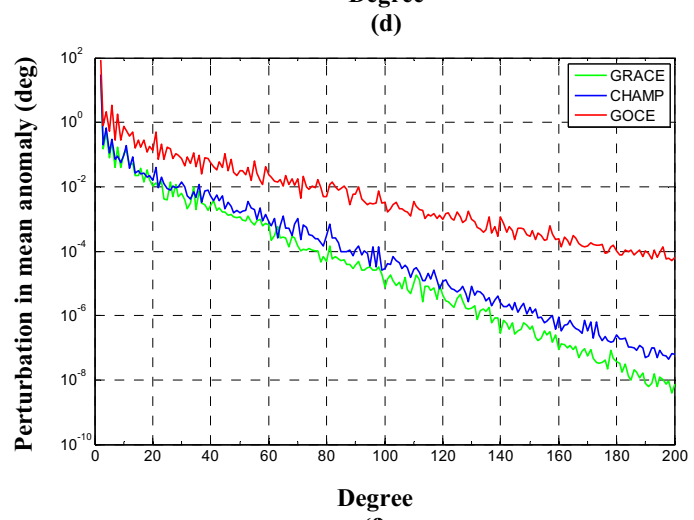

(f)

Fig.1. Absolute maximum value of geopotential perturbation in one day revolution of satellites, (a) perturbation in semi-major axis, (b) perturbation in eccentricity, (c) inclinations, (d) right ascension of ascending node, (e) perigee argument, (f) mean anomaly

Figure 1(a) shows the semi-major axis of the orbital ellipses of the recent satellite missions. The behaviour of the semi-major axis of the CHAMP and GRACE satellites are more or less the same as would be expected because of having close altitudes. However, in Fig. 1(a) one can see that the perturbation of these two satellites differs in some degrees. The perturbations of the CHAMP and the GRACE satellites in semimajor axis are nearly in the same order up to degree 50 but they differ in higher degrees. Because of lower altitude of the GOCE than the other satellites the semimajor axis of this satellite is more perturbed. It is perturbed more or less in the same order as the other satellites up to degree 30 but it differs. In order to see more details we can present the perturbations of the maximum degree on the orbital elements. A 
maximum degree could be considered for specific level of perturbation in each element. The millimeter level has been considered in the perturbation of semi-major axis and 1 arc-second for the angular elements. The maximum degree is 116 to see perturbations in millimeter level in semi-major axis of the CHAMP; this value is 109 and 175 for the GRACE and GOCE, respectively. The inclinations of the satellites are presented in Fig. 1(c). The figure shows the small perturbations in the inclination. They are in the same order for the lower degrees up to 20 and decreasing to 1 arcsecond. In Fig. 1(d) the large perturbations in the right ascension of ascending node are decreasing fast. They decrease to 1 arc-second up to degree 40 for the GOCE while it is about 20 for other satellites. The interesting matter is to see small perturbations on the satellite's orbit eccentricity, and this is why for dynamic orbit determination and analytical solution of the Earth gravitational field the satellite's orbit is assumed circular. It expresses that perturbation in eccentricity does not play an important role in orbit integration and recovery of the geopotential coefficients as the orbits are more or less circular. The behaviour of the perigee argument and mean anomaly is larger in the GOCE than the other satellites as it is expected. The perturbations in perigee arguments and mean anomaly are up to same degree 83 for the CHAMP and GRACE while these are considerably perturbed (twice larger) in GOCE.

In comparison with average power of accelerations, 154, 137 and 261 in these satellites missions, we can say that these values are too optimistic and theoretical, and the above numbers discussed are also too pessimistic for the maximum degree of perturbations. We expect that these differences are due to the selection of short arc orbit in this study. As it is known now, the maximum degree of the spherical harmonic expansion of the geopotential considered to be determined by the orbital analysis of CHAMP satellite is about 119 which does not confirm with the average power and the number that we obtained. The gravitational field extracted from the GRACE mission is expanded up to degree and order 150; but it should be kept in mind that satellite gradiometric data of the GRACE mission helped the solution to derive higher degrees and orders. Perturbation analysis of the satellite's orbit at altitude of the GRACE satellites cannot yield such resolution for the gravitational field from space. The maximum degree obtained using the average power of the acceleration for the GOCE is 261 but in this numerical study we obtain 175 based on short arc orbit consideration.

\section{CONCLUSIONS}

The newly presented expression for satellite acceleration computation in this paper is very simple for programming as it is not involved with the associated Legendre function derivatives and singular terms. However it should be mentioned that the singularity happens only for those satellites whose orbits inclined towards the poles. Since the GOCE orbit is away from the poles, the singularity is not needed to be considered. The coefficients of the associated Legendre functions in new expressions are constants and do not change by the position of a satellite and they should be computed once for all the integration process. The associated Legendre functions should be generated up to only two more harmonic degrees and orders in the new expression than in the traditional method. Another achievement is the capability of investigating perturbations of a satellite orbit parameters due to a single harmonic coefficient of the geopotential field during one day revolution of satellite. The average 
acceleration power was considered for the recent satellite's missions and the maximum degree of the spherical harmonic expansion are obtained 154, 137 and 261, for the CHAMP, GRACE and GOCE, respectively. The maximum absolute values of the perturbations of the orbital elements with respect to each degree of geopotential were computed by frequent integrating the satellites' orbit and comparing with Keplerian orbit. The perturbations were visualized in some figures presenting the behaviour of the orbital elements clearly. As we expected the perturbations of the CHAMP and GRACE satellite are similar and the GOCE orbit is perturbed considerably larger than the others. The maximum degrees of the spherical harmonic expansion of the orbit integration procedure was obtained based on a predefined amount of the perturbations (millimeter level in this study). This maximum values are 116, 109 and 175 for the CHAMP, GRACE and GOCE satellites, respectively, which contradicts with the degrees obtained from average acceleration power because we have considered one day revolution of satellite in our investigations.

\section{APPENDIX A}

Schematically we can write the system of differential equation as:

$x=\dot{x}$

$y=\dot{y}$

$z=\dot{z}$

$\ddot{x}=f_{4}(x, y, z, \Delta t)$

$\ddot{y}=f_{5}(x, y, z, \Delta t)$

$\ddot{z}=f_{6}(x, y, z, \Delta t)$

where, $\dot{x}, \dot{y}$ and $\dot{z}$ are the satellite's velocities in the quasi-inertial frame. The functions $f_{4}, f_{5}$ are the formulas presented in either Corollary 2 or 3 and $f_{6}$ is Corollary 1 . These equations can be solved by numerical integration algorithms. In this study we use 4-th order Runge-Kutta algorithm for solving the equation of motion of the satellite:

$x_{i+1}=x_{i}+\left(k_{11}+2 k_{21}+2 k_{31}+k_{41}\right) / 6$

$y_{i+1}=y_{i}+\left(k_{12}+2 k_{22}+2 k_{32}+k_{42}\right) / 6$

$z_{i+1}=z_{i}+\left(k_{13}+2 k_{23}+2 k_{33}+k_{43}\right) / 6$

$\dot{x}_{i+1}=\dot{x}_{i}+\left(k_{14}+2 k_{24}+2 k_{34}+k_{44}\right) / 6$

$\dot{y}_{i+1}=\dot{y}_{i}+\left(k_{15}+2 k_{25}+2 k_{35}+k_{45}\right) / 6$

$\dot{z}_{i+1}=\dot{z}_{i}+\left(k_{16}+2 k_{26}+2 k_{36}+k_{46}\right) / 6$

The coefficients of $k_{j k}, j=1,2,3,4$ and $k=1,2, \ldots, 6$ are presented. $i$ is the epoch number.

Generally, a higher order differential equations than first, is converted to a system of first order differential equations for the numerical solution; see Appendix B. In orbit integration the numerical solution of this system of differential equation yields the 
position and velocity of the satellite simultaneously in the next epoch of integration. For more detail see Appendix B.

The coefficient of 4-th order Runge-Kutta algorithm for solving the system of differential equation of satellite motion Eqs. (A1a)-(A1f)

$k_{11}=\dot{x}_{i} \Delta t_{i}$

$k_{12}=\dot{y}_{i} \Delta t_{i}$

$$
k_{21}=\left(\dot{x}_{i}+k_{14} / 2\right) \Delta t_{i}
$$

$k_{13}=\dot{z}_{i} \Delta t_{i}$

$k_{22}=\left(\dot{y}_{i}+k_{15} / 2\right) \Delta t_{i}$

$k_{14}=f_{4}\left(x_{i}, y_{i}, z_{i}\right) \Delta t_{i}$

$k_{23}=\left(\dot{z}_{i}+k_{16} / 2\right) \Delta t_{i}$

$k_{15}=f_{5}\left(x_{i}, y_{i}, z_{i}\right) \Delta t_{i}$

$k_{24}=f_{4}\left(x_{i}+k_{11} / 2, y_{i}+k_{12} / 2, z_{i}+k_{13} / 2\right) \Delta t_{i}$

$k_{16}=f_{6}\left(x_{i}, y_{i}, z_{i}\right) \Delta t_{i}$,

$k_{25}=f_{5}\left(x_{i}+k_{11} / 2, y_{i}+k_{12} / 2, z_{i}+k_{13} / 2\right) \Delta t_{i}$

$k_{26}=f_{6}\left(x_{i}+k_{11} / 2, y_{i}+k_{12} / 2, z_{i}+k_{13} / 2\right) \Delta t_{i}$

$k_{31}=\left(\dot{x}_{i}+k_{24} / 2\right) \Delta t_{i}$

$k_{41}=\left(\dot{x}_{i}+k_{34}\right) \Delta t_{i}$

$k_{32}=\left(\dot{y}_{i}+k_{25} / 2\right) \Delta t_{i}$

$k_{42}=\left(\dot{y}_{i}+k_{35}\right) \Delta t_{i}$

$k_{33}=\left(\dot{z}_{i}+k_{26} / 2\right) \Delta t_{i}$

$k_{43}=\left(\dot{z}_{i}+k_{36}\right) \Delta t_{i}$

$k_{34}=f_{4}\left(x_{i}+k_{21} / 2, y_{i}+k_{22} / 2, z_{i}+k_{23} / 2\right) \Delta t_{i} \quad k_{44}=f_{4}\left(x_{i}+k_{31}, y_{i}+k_{32}, z_{i}+k_{33}\right) \Delta t_{i}$

$k_{35}=f_{5}\left(x_{i}+k_{21} / 2, y_{i}+k_{22} / 2, z_{i}+k_{23} / 2\right) \Delta t_{i} \quad k_{45}=f_{5}\left(x_{i}+k_{31}, y_{i}+k_{32}, z_{i}+k_{33}\right) \Delta t_{i}$

$k_{36}=f_{6}\left(x_{i}+k_{21} / 2, y_{i}+k_{22} / 2, z_{i}+k_{23} / 2\right) \Delta t_{i} \quad k_{46}=f_{6}\left(x_{i}+k_{31}, y_{i}+k_{32}, z_{i}+k_{33}\right) \Delta t_{i}$

$x_{i}, y_{i}, z_{i}, \dot{x}_{i}, \dot{y}_{i}$ and $\dot{z}_{i}$ are the three components of the position and velocity at reference epoch i, respectively. $\Delta t_{i}$ is the integration step size.

\section{APPENDIX B}

The solution of the higher order differential equation leads to a system of first order differential equations and this system of differential equations can numerically be solved. For an m-order differential equation we write

$y=y(t), a \leq t \leq b$

is the solution of the following m-order differential equation

$y^{(m)}(t)=f\left(y, y^{\prime}, \cdots, y^{(m-1)}, t\right)$

with the following initial values

$y\left(t_{0}\right)=\alpha_{1}, y^{\prime}\left(t_{0}\right)=\alpha_{2}, \cdots, y^{(m-1)}\left(t_{0}\right)=\alpha_{m}$.

A system of first order differential equations can be written:

$y(t)=v_{1}(t), \quad y^{\prime}(t)=v_{2}(t), \ldots, y^{(m-1)}(t)=v_{m}(t)$

and 
$\left\{\begin{array}{l}\frac{d v_{1}}{d t}=\frac{d y}{d t}=v_{2} \\ \frac{d v_{2}}{d t}=\frac{d y^{\prime}}{d t}=v_{3} \\ \vdots \\ \frac{d v_{m}}{d t}=\frac{d y^{(m-1)}}{d t}=y^{(m)}=f\left(y, y^{\prime}, \cdots, y^{(m-1)}, t\right) \\ =f\left(v_{1}, v_{2}, \cdots, v_{m}, t\right)\end{array}\right.$

is the system of $\mathrm{m}$ first order differential equations with the following initial values

$$
\begin{aligned}
& v_{1}\left(t_{0}\right)=y\left(t_{0}\right)=\alpha_{1} \\
& v_{2}\left(t_{0}\right)=y^{\prime}\left(t_{0}\right)=\alpha_{2} \\
& \vdots \\
& v_{m}\left(t_{0}\right)=y^{(m-1)}\left(t_{0}\right)=\alpha_{m}
\end{aligned}
$$

The numerical solution of the system of differential equations by for example RungeKutta is

$$
\begin{aligned}
& y_{n}=y\left(t_{n}\right), \text { and } \quad z_{n}=z\left(t_{n}\right), \\
& y^{\prime}=f(y, z, t), \text { and } \quad z^{\prime}=g(y, z, t),
\end{aligned}
$$

and coefficients of the Runge-Kutta algorithm are:

$$
\begin{aligned}
& k_{1}=f\left(y_{i}, z_{i}, t_{i}\right) \Delta t_{i} \\
& m_{1}=g\left(y_{i}, z_{i}, t_{i}\right) \Delta t_{i} \\
& k_{2}=f\left(y_{i}+k_{1} / 2, z_{i}+m_{1} / 2, t_{i}+\Delta t_{i} / 2\right) \Delta t_{i} \\
& m_{2}=g\left(y_{i}+k_{1} / 2, z_{i}+m_{1} / 2, x_{i}+\Delta t_{i} / 2\right) \Delta t_{i} \\
& k_{3}=f\left(y_{i}+k_{2} / 2, z_{i}+m_{2} / 2, t_{i}+\Delta t_{i} / 2\right) \Delta t_{i} \\
& m_{3}=g\left(y_{i}+k_{2} / 2, z_{i}+m_{2} / 2, t_{i}+\Delta t_{i} / 2\right) \Delta t_{i} \\
& k_{4}=f\left(y_{i}+k_{3}, z_{i}+m_{3}, t_{i}+\Delta t_{i}\right) \Delta t_{i} \\
& m_{4}=g\left(y_{i}+k_{3}, z_{i}+m_{3}, t_{i}+\Delta t_{i}\right) \Delta t_{i}
\end{aligned}
$$

and the differential equations are numerical solved by the following relations

$$
\begin{aligned}
& y_{i+1}=y_{i}+\left(k_{1}+2 k_{2}+2 k_{3}+k_{4}\right) / 6 \\
& z_{i+1}=z_{i}+\left(m_{1}+2 m_{2}+2 m_{3}+m_{4}\right) / 6 .
\end{aligned}
$$

Acknowledgments. The authors would like to thank Prof. Lars E. Sjöberg for his valuable comments on the draft version of this paper. The first author appreciates the Swedish National Space Board for the financial support, project no. 63/07:1. 


\section{REFERENCES}

Albertella A., Migliaccio F., and Sansò F. (2002) GOCE: The Earth Field by Space Gradiometry. Celestial Mechanics and Dynamical Astronomy, Vol. 83, 1-15.

Babolian E. and Malek Nejad K. (1994) Numerical computations, University of Elm va San'at publication, Tehran, Iran.

Balmino G., Perosanz F., Rummel R., Sneeuw N., Sünkel H. and Woodworth P., (1998) European Views on Dedicated Gravity Field Missions: GRACE and GOCE. An Earth Sciences Division Consultation Document, ESA, ESD-MAG-REP-CON001.

Balmino G., Perosanz F., Rummel R., Sneeuw N. and Suenkel H. (2001) CHAMP, GRACE and GOCE: Mission Concepts and Simulations. Boll. Geof. Teor. Appl., Vol. 40, No. 3-4, 309-320.

ESA (1999) Gravity Field and Steady-State Ocean Circulation Mission, ESA SP1233(1), Report for mission selection of the four candidate earth explorer missions. ESA Publications Division, pp. 217, July 1999.

Eshagh M. (2003a) Precise orbit determination of a low Earth orbiting satellite, MSc thesis, K. N. Toosi University of Technology, Tehran, Iran.

Eshagh M. (2003b) Consideration of the effect of Gravitational and non-gravitational forces acting on a low Earth orbiting satellite, paper to be presented in 11-th National Iranian Geophysical Conference, 1-3 December, National Geological Center, Tehran, Iran.

Eshagh M. (2005) Step variable numerical orbit integration of a low Earth orbiting satellite, Journal of the Earth \& Space Physics, Vol. 31, No. 1, 1-12.

Eshagh, M., and Najafi Alamdari, M. (2005a) Investigation of orbital perturbations of a low Earth orbiting (LEO) satellite, paper to be presented in NATM June 27-29, Institute of Navigation ION 61 St. Cambridge, Massachusetts, United States of America.

Eshagh M. and Najafi-Alamdari M. (2005b) Numerical orbit integration of a low Earth orbiting satellite, paper to be presented in European navigation conference, GNSS 2005, German Institute of Navigation, Muenchen, Germany.

Eshagh M. and Najafi-Alamdari M. (2006) Comparison of different numerical integration methods of orbit integration, Journal of the Earth \& Space Physics. Vol. 33, No. 1, 41-57. (in Persian)

Eshagh M. and Najafi-Alamdari M. (2007) Perturbations in orbital elements of a low Earth orbiting satellite, Journal of the Earth \& Space Physics. Vol. 33, No. 1, 1-12.

Eshagh M. (2008a) Non-singular expression for the vector and gradient tensor of gravitation in a geocentric spherical frame, Computers \& Geosciences, Vol. 34, 1762-1768.

Eshagh M. (2009a) Impact of vectorization in global synthesis and analysis in gradiometry, Acta Geodaetica et Geophysica Hungarica (Accepted)

Eshagh M. (2009b) Orbit integration in non-inertial frames, Journal of the Earth \& Space Physics. (Accepted)

Heiskanen W.A. and Moritz H. (1967) Physical geodesy, W.H. Freeman and Company

Hwang C. and Lin J.M. (1998) Fast integration of low orbiter's trajectory perturbed by the earth's non-sphericity, Journal of Geodesy, Vol. 72, 578-585

Ilk K.H. (1983) Ein eitrag zur Dynamik ausgedehnter KörperGravitationswechselwirkung. Deutsche Geodätische Kommission. Reihe C, Heft Nr. 288, München, $181 \mathrm{pp}$.

Kaula W. (1966) Theory of satellite geodesy, Blaisdell, Waltheim 
Keller W. and Sharifi M. A. (2005) Satellite gradiometry using a satellite pair. Journal of Geodesy, Vol. 78, 544-557,

Lemoine F.G., Kenyon S.C., Factor J.K., Trimmer R.G., Pavlis N.K., Chinn D.S., Cox C.M., Klosko S.M., Luthcke S.B., Torrence M.H., Wang Y.M., Williamson R.G., Pavlis E.C., Rapp R.H. and Olson T.R. (1998) The Development of the Joint NASA GSFC and NIMA Geopotential Model EGM96, NASA/TP-1998-206861. Goddard Space Flight Center, Greenbelt

Parrot D. (1989) Short arc orbit improvement for GPS satellites, MSc thesis, Department of Surveying Engineering, University of New Brunswick, Canada.

Reigber C., Schwintzer P. and Lühr H. (1999) The CHAMP geopotential mission, Boll. Geof. Teor. Appl. Vol. 40, 285-289.

Reigber Ch., Jochmann H., Wünsch J., Petrovic S., Schwintzer P., Barthelmes F., Neumayer K.-H., König R., Förste Ch., Balmino G., Biancale R., Lemoine J.-M., Loyer S. and Perosanz F. (2004) Earth Gravity Field and Seasonal Variability from CHAMP. In: Reigber, Ch., Lühr, H., Schwintzer, P., Wickert, J. (eds.), Earth Observation with CHAMP - Results from Three Years in Orbit, Springer, Berlin, 25-30.

Rim H. J. and Schutz B. E. (2001) Precision orbit determination (POD), Geoscience laser and altimeter satellite system, University of Texas, United States of America.

Rummel R., Sanso F., Gelderen M., Koop R., Schrama E., Brovelli M., Migiliaccio F., and Sacerdote F. (1993) Spherical harmonic analysis of satellite gradiometry. Publ Geodesy, New Series, No. 39 Netherlands Geodetic Commission, Delft

Santos M. C. (1994) On real time orbit improvement for GPS satellites, Ph.D thesis, Department of Geodesy and Geomatics Engineering, University of New Brunswick, Canada.

Sharifi M.A. (2006) Satellite to satellite tracking in the space-wise approach, $\mathrm{PhD}$ dissertation, Geodätisches Institut der Universität Stuttgart.

Sneeuw N. (1992) Representation coefficients and their use in satellite geodesy, Manuscripta Geodaetica, Vol. 17, 117-123.

Su H. (2000) Orbit determination of IGSO, GEO and MEO satellites, Ph.D thesis, Department of Geodesy, University of Bundeswehr, Munchen, Germany

Tapley B., Ries J. Bettadpur S., Chambers D., Cheng M., Condi F., Gunter B., Kang Z., Nagel P., Pastor R., Pekker T., Poole S. and Wang F. (2005) GGM02-An improved Earth gravity field model from GRACE. Journal of Geodesy, Vol. 79, 467-478.

Visser P. (1992) The use of satellites in gravity field determination and adjustment, $\mathrm{PhD}$ dissertation, University of Delft

Wolf R. (2000) Satellite orbit and ephemeris determination using inter satellite links, Ph.D thesis, Department of Geodesy, University of Bundeswehr, Munchen, Germany.

Received: 2008-11-27,

Reviewed: 2009-03-05,

Accepted: 2009-03-09. 